\title{
Editorial
}

Pathobiology

Published online: November 30, 2017

\section{Next-Generation Sequencing in Diagnostics and Clinical Research}

\author{
Gerald Hoefler \\ Medical University of Graz, Graz, Austria
}

Data obtained from analyzing the tumor genome using next-generation sequencing (NGS) technologies have revolutionized our understanding of tumor biology and paved the way to personalized medicine in oncology.

It is hard to believe, as Stenzinger and Weichert [this issue, pp. 306-322] observe, that the first human genomes have been sequenced only about 15 years ago. Today, whole-exome or whole-genome data from thousands of tumors have been published in peer-reviewed journals and even more have been deposited in public databanks. We know now that typical tumors contain 2-8 "driver gene" mutations that convey a growth advantage and up to 100 "passenger gene" mutations. Mutations in driver genes affect signaling pathways that regulate core cellular processes such as cell fate, cell survival, and genome maintenance. The actual number of mutations varies between tumors, from low numbers in pediatric tumors to tumors that are associated with potent mutagen such as adenocarcinoma of the lung (cigarette smoke) and melanoma (ultraviolet radiation) that typically contain over 100 mutations. Tumors with DNA mismatch repair defects are an exception with $>1,000$ mutations. According to the prevalent view on tumor development, a gatekeeping mutation is followed by series of clonal tumor cell

\section{KARGER}

๑) 2017 S. Karger AG, Basel

E-Mail karger@karger.com

www.karger.com/pat expansions that are associated with driver gene mutations providing additional growth advantage. Astonishingly, more than $50 \%$ of passenger mutations are thought to occur at the preneoplastic phase leading to the concept that a large proportion of these mutations are random events present in a cell before the initiating event in tumorigenesis takes place. Of further note, almost all mutations of metastatic cancer are already present in the primary tumor. Sequencing data from different tumor areas as well as metastases have also greatly improved our understanding of tumor heterogeneity. Detecting shedded tumor cells in peripheral blood and especially shedded tumor DNA in serum is already a valuable test to monitor therapy efficacy in tumor patients. Its importance will increase in the near future and it will be a very valuable tool to design optimal therapeutic approaches.

As Stenzinger and Weichert [this issue, pp. 306-322] stress, translating this enormous increase in knowledge into clinically important tests was pioneering work triggered by observations that were made more than 10 years ago showing that patients with KRAS-mutant colorectal carcinoma (CRC) do not respond to EGFR targeting agents. These data led to a major change in the approval of these drugs by the European Medical Agency (EMA), 
which in 2008 demanded genetic testing of CRC for the presence of KRAS mutations as a prerequisite for antibody treatment. Initially, many institutions focused on Sanger sequencing or other methods for hot-spot mutation detection. Many labs now have switched to NGS using customized multigene panels since sequencing of whole exomes or even whole genomes in high-throughput routine diagnostics was (and still is) neither cost-effective nor efficient in terms of turnaround time.

In this issue, Ilyas [pp. 292-305] provides an overview on the challenges pathologists face when implementing NGS in diagnostic pathology. He stresses that molecular data need to be interpreted and integrated with the morphological data and that the pathologist must understand the utility as well as the limitations of NGS data. Importantly, a scheme is presented to "classify" the types of data which are generated. In addition, a glossary of the new terminology is included to help pathologists become familiar with the lexicon of NGS-derived molecular data.

Stenzinger and Weichert [this issue, pp. 306-322] not only present an excellent overview on genetic profiling of cancers of the digestive system, focusing on biological insights and clinical implications, but also raise many thought-provoking issues. An illustrative example is CRC, in which 4 genetically distinct groups are also reflected by different morphological characteristics and clinical features: (1) non-hypermutated CRC with chromosomal instability (CIN) driven by mutations in KRAS as well as loss of heterozygosity in the tumor suppressor genes APC and TP53; (2) hypermutated CRC with defective mismatch repair genes resulting in microsatellite instability and tumors with mutations affecting the proofreading domain of the DNA polymerase POLE; (3) CRC characterized by the $\mathrm{CpG}$ island methylator phenotype (CIMP); and (4) hypermutated and non-hypermutated cancers with genetic alterations in tetranucleotide repeats of microsatellites. Most importantly, this classification will lead to treatment implications. Microsatellite instable CRC respond well to anti-EGFR treatment and, presumably, by the appearance of novel immunogenic neoantigens, are a prime target for therapy with immune checkpoint inhibitors.

The picture is less clear for other types of cancers of the digestive system. A novel recurring theme seems to be mutations in chromatin-remodeling factors, especially in the ARID1A and ARID2 genes that have been found to be frequently mutated in esophageal adenocarcinoma and already in nondysplastic Barrett's esophagus indicating very early events in cancerogenesis. Mutations in other genes such as TP53 are confined to later stages (high- grade dysplasia and invasive carcinoma). Therefore, interrogation of these genes might be suitable to supplement conventional histology in the assessment of risk progression. Frequent inactivating mutations in the ARID1A and ARID2 genes are also found in gastric cancer, most prevalent in MSI-driven tumors, closely followed by tumors associated with EBV infections. Mutations in these genes are also prevalent in hepatocellular cancer as well as in cholangiocarcinoma.

Another very important lesson that can be drawn from CRC is the fact that mutations in founder genes are already apparent early in the development of colorectal neoplasms including clinically benign precursor lesions (e.g., frequent BRAF mutations in pre-invasive lesions of the serrated pathway including hyperplastic polyps). Therefore, mutation detection as a single parameter without taking into account the biological and morphological context - is non-informative and could be even misleading if diagnostic and therapeutic decisions were to be solely based on this marker.

Szurian et al. [this issue, pp. 323-338] provide an excellent overview on the role of NGS as a diagnostic tool for the evaluation of bone and soft-tissue tumors. This is especially valuable for pathologists involved in the diagnostics of these in general rare tumors that nevertheless comprise more than 117 different soft-tissue tumor and 58 bone tumor disease entities as defined in the recent WHO classification of bone and soft-tissue tumors. Considering that more than half of the described soft-tissue tumors and approximately $25 \%$ of the bone tumors harbor recurrent genetic alterations, the use of auxiliary molecular examinations should be strongly considered since diagnosing these tumors is challenging based on the large number of different tumor entities, the rareness of these tumors, and the considerable morphological variations.

Based on their experience, the authors regard NGS using the Archer FusionPlex Sarcoma Kit to be superior to fluorescent in situ hybridization as an auxiliary tool in the routine workup of soft-tissue and bone tumors. This is evidenced by several examples illustrating the use of this technique. For example, the differential diagnosis between chondroblastoma and giant cell tumor of the bone is aided by the fact that $\mathrm{K} 36 \mathrm{M}$ mutations in the $H 3 F 3 B$ gene are found in $95 \%$ of chondroblastomas, whereas H3F3A mutations are prevalent in $92 \%$ of giant cell tumors of the bone. Both genes encode for histone H3.3, an intriguing fact, regarding the pathogenesis of these tumors. The identification of Ewing sarcoma and Ewinglike sarcoma is aided by specific translocations present in those tumors that involve the EWSR1 as well as the FUS 
gene in conjunction with various specific partners in Ewing sarcoma and the EWSR $1, C I C$, and BCOR genes, again with various specific translocation partners, in Ewinglike sarcoma. In addition to its use as a diagnostic tool, NGS may detect the presence of mutations and translocations, which are potential targets of molecular-based personalized treatment options in Ewing sarcoma or desmoplastic small round cell tumor. These include tumors with mutations in the PTPRD, GRB10, and KRAS genes for which IGF1R inhibitor treatment in combinations with cixutumumab and mTOR inhibitor for Ewing sarcoma, Ewing-like sarcoma as well as desmoplastic small round cell tumor can be considered. This excellent review offers high-quality illustrations as well as tables providing a comprehensive overview on differential diagnostic guidelines of bone and soft-tissue neoplasms including vascular and myxoid neoplasms according to their genetic alterations and the therapeutic relevance.

A different and indeed very intriguing approach is chosen by van Winkel et al. [this issue, pp. 339-350] to investigate the prognostic and predictive value of the most common mutations found in ovarian, endometrial, and cervical tumors. After a critical appraisal of the literature using the "Quality in Prognostic Studies" tool, data on mutation incidence and correlations with prognostic and predictive items were extracted from relevant studies and compared to their own cohort consisting of 28 patients analyzed using NGS. Eight out of 739 articles were found to be eligible and clinical data were available for 17 of their patients. Based on these data, KRAS mutations were found to be associated with type I ovarian tumors (low-grade serous, mucinous, endometrioid, and clear-cell) and seem to have a more favorable prognosis.
Surprisingly, the prognostic value of TP53 remains controversial. In endometrial tumors, PTEN mutations show a positive correlation with better prognosis, whereas PIK3CA may have a correlation with poorer prognosis. CTNNB1 mutations in endometrial carcinomas could predict a worse prognosis.

The future of NGS testing is touched upon by Stenzinger and Weichert [this issue, pp. 306-322]. They predict that if only a fraction of the current clinical studies would be successful, the approach to testing will have to broaden tremendously with respect to both specific markers and the amount and types of cancers that require testing. Moreover, whole-exome and whole-genome sequence data could potentially be used to provide unbiased tumor mutational landscapes during routine diagnostics using comprehensive analyses of data sets. This will lead to the discovery of the true prevalence of less frequent genetic aberrations in the intention-to-treat population but also to the detection of novel mutational patterns in subsets of major cancers as well as in rare tumors that may reshape diagnostic categories. The cooperation of centers on an international level to establish a comprehensive database on mutations in all kinds of tumors including clinical data would be a major accomplishment.

As the number of "druggable" mutations has greatly increased in the last few years, it is now obligatory to know the mutational status of target genes before targeted treatment can start for the majority of malignant tumors (e.g., colon cancer, adenocarcinoma of the lung, malignant melanoma, GIST). The enormous potential of NGS is now finding its way into daily diagnostic practice in molecular pathology as well as in the clinic. 